\title{
La protection de l'accès au juge judiciaire par les normes fondamentales
}

The protection of the right of access to a judge by fundamental norms

\section{Antoine Steff}

\section{(2) OpenEdition}

\section{Journals}

Édition électronique

URL : http://journals.openedition.org/add/561

DOI : $10.4000 /$ add. 561

ISSN : 2606-1988

\section{Éditeur}

Presses universitaires de Rouen et du Havre

\section{Édition imprimée}

Date de publication : 16 octobre 2017

Pagination : 233-253

ISBN : 979-10-240-0775-5

ISSN : 1955-0855

\section{Référence électronique}

Antoine Steff, "La protection de l'accès au juge judiciaire par les normes fondamentales », Les Annales de droit [En ligne], 11 | 2017, mis en ligne le 16 octobre 2018, consulté le 23 avril 2019. URL : http:// journals.openedition.org/add/561; DOI : 10.4000/add.561 


\section{La protection de l'accès au juge judiciaire par les normes fondamentales}

Antoine STEFF

Le droit d'accès à un tribunal ou encore le droit à un recours juridictionnel ou enfin le droit à un juge, a pu être défini comme «le droit pour toute personne physique ou morale, française ou étrangère, d'accéder à la justice pour y faire valoir ses droits ${ }^{1}$ ».

Quoique tout vestige de la justice privée n'ait pas totalement disparu en droit positif, ce principe du libre accès aux tribunaux apparait, dans un État de droit, comme la contrepartie de l'interdit de la vengeance privée exprimé par l'adage "Nul ne peut se faire justice à soi-même", au fondement du contrat social, et dont les origines remonteraient au droit romain ${ }^{2}$.

Plus près de nous, comme l'a écrit le grand processualiste italien, Giuseppe Chiovenda, « le fait d'interdire à quiconque de se faire justice à soi-même a pour corollaire indispensable la faculté reconnue à tous, sans discrimination d'aucune sorte, de saisir les tribunaux en vue d'obtenir justice ${ }^{3} »$.

Entendu d'abord comme une liberté publique ${ }^{4}$, l'accès à un juge est librement et également ouvert à toute personne. Peu importe que la demande du plaideur soit ou non, recevable, régulière ou bien fondée. Sous peine de déni de justice, le juge devra répondre à cette demande, au

1. Louis Favoreu et Thierry-Serge Renoux, «Le contentieux constitutionnel des actes administratifs», extrait du Répertoire Dalloz du contentieux administratif, Paris, Sirey, 1992, p. 90 et suiv.

2. Serge Guinchard, Procédure civile, Paris, Dalloz, 2012, p. 11 ; Travaux de l'association Henri-Capitant, "Nul ne peut se faire justice à soi-même : le principe et ses limites ", Journées françaises de Lyon, Grenoble et Aix-en-Provence, RID comp. 1967, vol. 19, p. 938.

3. Giuseppe Chiovenda, Istituzioni di diritto processuale civile, Naples, 1933, vol. 1, $\mathrm{n}^{\mathrm{o}}$ 12, cité par Serge Guinchard, Procédure civile, op. cit.

4. Loïc Cadiet, Théorie générale du procès, Paris, PUF, 2010, $\mathrm{n}^{\circ} 78$, p. 315 ; Serge Guinchard, Procédure civile, op. cit. 
besoin en se déclarant incompétent, ou bien en la déclarant irrecevable ou encore en en constatant l'irrégularité.

Le droit d'accès à un tribunal ne se confond pas avec l'action en justice. Consacrée, après sa longue transformation la distinguant progressivement, à la différence du droit romain, du droit substantiel dont elle assure la sanction ${ }^{5}$, comme la faculté générale et reconnue par la puissance publique de faire protéger des droits subjectifs par un juge ${ }^{6}$, elle est le prolongement ou l'expression directe du droit d'accès à un tribunal ${ }^{7}$. «Le droit pour l'auteur d'une prétention d'être entendu sur le fond de celle-ci» qui, aux termes de l'article 30 du Code de procédure civile, définit l'action en justice, suppose préalablement garanti, en effet, le droit pour le plaideur de saisir le juge, d'accéder au tribunal et de déclencher son fonctionnement. Cependant, ces dispositions de l'article 30 et des suivants du Code de procédure civile qui définissent l'action comme un droit et énoncent certaines des conditions auxquelles son exercice est subordonné, ne la protègent aucunement contre les atteintes ou restrictions de toute sorte qu'elle peut subir du pouvoir normatif ou d'autres sources ${ }^{8}$.

Or, c'est précisément le caractère fondamental, reconnu relativement récemment au droit d'accès à un tribunal, qui vient en protéger et en encadrer l'exercice.

Le droit d'accès au juge ne se confond pas davantage avec le droit plus général à un recours, dont il est l'une des expressions ${ }^{9}$. Le droit à un recours peut, en effet, en dehors du procès pénal, ne pas être juridictionnel. Il est plus large que le droit d'accès à un tribunal et comprend, en droit français, le droit à un recours administratif, gracieux ou hiérarchique. Cette distinction est d'ailleurs également présente en droit européen des droits de l'homme ${ }^{10}$, comme en droit international ${ }^{11}$.

5. Serge Guinchard, Procédure civile, op. cit., $\mathrm{n}^{\circ} 82$, p. 113.

6. Joseph Pini et Thierry-Serge Renoux, Dictionnaire des droits fondamentaux, Paris, Dalloz, 2007, p. 503.

7. Serge Guinchard, Procédure civile, op. cit., $\mathrm{n}^{\circ} 81, \mathrm{p} .113$.

8. Loïc Cadiet, Théorie générale du procès, op. cit.

9. Serge Guinchard, Droit processuel. Droits fondamentaux du procès, Paris, Dalloz, $2011, \mathrm{n}^{0} 234$ et suiv., p. 508.

10. Voir les dispositions des articles 6 et 13 de la convention européenne de sauvegarde des droits de l'homme et des libertés fondamentales.

11. Voir le pacte international relatif aux droits civils et politiques du 19 décembre 1966. 
Quant à sa place dans la hiérarchie des normes, en droit interne français, contrairement à d'autres constitutions européennes ${ }^{12}$, la constitution ne contient pas de disposition expresse garantissant l'accès au juge.

Certes, une doctrine constitutionnaliste a soutenu son existence et sa valeur constitutionnelle sur le fondement de principes écrits et non écrits ${ }^{13}$ et il a, par ailleurs, été reconnu par les instruments internationaux universels ${ }^{14}$. Mais c'est la Cour européenne de sauvegarde des droits de l'homme et des libertés fondamentales (CEDH) qui, la première, dans son célèbre arrêt Golder c/Royaume-Uni du 21 février $1975^{15}$, a affirmé et sanctionné le droit à un tribunal comme l'un des piliers du droit au procès équitable garanti par l'article $6 \$ 1$ de la convention, tout en énonçant dès cette décision que ce droit n'est pas absolu et peut faire l'objet de limitations. L'arrêt Deweer c/Belgique du 27 février 1980 a confirmé cette jurisprudence en matière pénale ${ }^{16}$.

Par une interprétation «dynamique» faisant appel à la théorie des «éléments inhérents à un droit », «reconstruisant » ainsi le contenu du droit à un procès équitable, la Cour européenne a ajouté aux garanties procédurales stricto sensu énoncées expressément ${ }^{17}$ ou implicitement ${ }^{18}$

12. Joseph Pini et Thierry-Serge Renoux, op. cit., p. 505, qui évoquent à cet égard les constitutions espagnole, italienne et allemande.

13. Serge Guinchard, Procédure civile, op. cit, $\mathrm{n}^{0}$ 109, p. 134 et Droit processuel. Droits fondamentaux du procès, op. cit., $\mathrm{n}^{\circ} 247, \mathrm{p} .541$, qui évoque en particulier à cet égard, respectivement, le principe de la séparation des pouvoirs et l'interdiction du déni de justice.

14. Voir respectivement les articles 8 de la déclaration universelle des droits de l'homme et $14 \$ 12^{\circ}$ du pacte international relatif aux droits civils et politiques du 19 décembre 1966.

15. Après avoir relevé $(\$ 34)$ que «la prééminence du droit ne se conçoit guère sans la possibilité d'accéder aux tribunaux», la Cour a énoncé $(\$ 35)$ qu' «on ne comprendrait pas que l'article $6 \S 1^{\mathrm{er}}$ décrive en détail les garanties de procédure accordées aux parties à une action civile en cours et qu'il ne protège pas d'abord ce qui seul permet d'en bénéficier en réalité: l'accès au juge». "Équité, publicité, célérité du procès n'offrent point d'intérêt en l'absence de procès ", puis a affirmé que «le droit d'accès constitue un élément inhérent au droit qu'énonce l'article $6 ₫ 1$ ». Et la Cour de conclure en l'espèce qu' «en répondant qu'il ne croyait pas devoir accorder la permission sollicitée, le ministre a méconnu dans la personne du requérant le droit de saisir un tribunal, tel que le garantit l'article $6 \$ 1$ », CEDH, 21 févr. 1975, Golder c/R-U, série A n ${ }^{\circ} 18$.

16. $\mathrm{CEDH}, 27$ févr. 1980, Deweer c/Belgique, série $\mathrm{A} \mathrm{n}^{\circ} 35$.

17. I. e. les principes d'indépendance et d'impartialité du tribunal, de publicité et de célérité de la procédure.

18. I.e. le principe d'égalité des armes. 
par le texte ${ }^{19}$, le droit d'accès à un tribunal de même que le droit à l'exécution des décisions de justice.

Cette consécration du droit d'accès à un tribunal en tant que droit fondamental peut s'entendre à un double titre: substantiel, par le caractère essentiel du principe de la prééminence du droit qu'il promeut, formel, ensuite, en raison de la place qu'il occupe dans la hiérarchie des normes. Il a ainsi la même valeur supra-législative que l'ensemble des règles et principes qui découlent de la convention, et s'impose, à ce titre, à toutes les autorités des États contractants avec une force supérieure à celle de leurs propres lois.

La Cour de justice de Luxembourg, dans le sillage de la Cour européenne, a également reconnu ce droit, tout d'abord au titre des principes généraux du droit communautaire, en se fondant sur les traditions constitutionnelles communes des États membres et les articles 6 et 13 de la $\mathrm{CEDH}^{20}$ puis, par la suite, sur la charte des droits fondamentaux de l'Union (article 47), qui garantit le recours effectif à un juge et non pas seulement à une instance nationale ${ }^{21}$. Un recours juridictionnel doit donc être accordé «même si les règles de la procédure interne ne le prévoient pas $^{22} »$.

Le Conseil constitutionnel français, à l'issue d'une construction très progressive de sa jurisprudence ${ }^{23}$, a finalement affirmé directement, sur le fondement de l'article 16 de la Déclaration des droits de l'homme et du citoyen de 1789 (DDHC), le caractère constitutionnel du droit à un recours juridictionnel effectif, tout en considérant qu'il puisse faire l'objet d'atteintes, pourvu qu'elles ne soient pas substantielles ${ }^{24}$.

19. Frédéric Sudre, Droit européen et international des droits de l'homme, Paris, PUF, $2012, n^{\circ} 211$, p. 373.

20. CJCE, 15 mai 1986, Margaret Johnston, aff. 222/84, Rec., 1986, p. 1651.

21. CJCE, 13 mars 2007, Unibet, C-432/05, Rec., 2007 p. I-2271.

22. CJCE, 3 déc. 1992, Oleificio Borelli c/Commission, C-97/91, Rec., 1992, p. I-6313.

23. Voir la décision du Conseil constitutionnel no 93-335 DC du 21 janvier 1994 (considérant 4) qui rattache le droit d'exercer un recours (en l'espèce l'exception d'illégalité devant le juge administratif) à l'article 16 de la DDHC de 1789 et assure ainsi la garantie des droits.

24. Voir la décision du Conseil constitutionnel $n^{\circ}$ 96-373 DC du 9 avril 1996, maintes fois confirmée par la suite, qui énonce que «la loi ne saurait porter d'atteintes substantielles au droit des intéressés d'exercer un recours effectif devant une juridiction »; voir Joseph Pini et Thierry-Serge Renoux (op. cit., n. 6), relevant que le Conseil constitutionnel a ainsi affirmé directement la reconnaissance du caractère constitutionnel du droit à un recours juridictionnel effectif en le rattachant explicitement à l'idée de garantie des droits, telle qu'elle ressort de l'article 16 de la DDHC du 26 août 1789 , et a également affirmé la nécessité générale d'assurer une protection efficace des droits proclamés, en liant celle-ci à l'idée même de 
La loi $\mathrm{n}^{0}$ 98-657 du 29 juillet 1998 relative à la lutte contre les exclusions a, par ailleurs, consacré en son article $1^{\text {er }}$ un droit fondamental d'accès à la justice en ce qu'elle "tend à garantir sur l'ensemble du territoire l'accès effectif de tous aux droits fondamentaux dans les domaines... de la justice».

Ce caractère fondamental du droit au juge a aussi été accueilli progressivement tant par la juridiction administrative ${ }^{25}$ que par le juge judiciaire ${ }^{26}$.

constitution : «La garantie des droits d'une part est constituée d'éléments extérieurs (et postérieurs) à la DDHC elle-même (par exemple la compétence législative en matière de "garanties fondamentales pour l'exercice des libertés publiques", selon l'article 34 de la Constitution ou la jurisprudence relative à "l'incompétence négative"), d'autre part dépasse la seule protection du droit d'exercer un recours juridictionnel effectif et est souvent assimilée au principe de légalité ou, plus largement, à la hiérarchie des normes protectrices des droits proclamés et protégée par le juge.»

25. Devant le juge administratif le droit «à un juge de [...] tout individu en face de l'arbitraire toujours possible du pouvoir" a fait l'objet d'une reconnaissance ancienne: voir conclusions Henry sur CE, 11 mai 1962, Salan, RDP 1962, p. 549. Dès le début du XIX ${ }^{\mathrm{e}}$ siècle le Conseil d'État a admis des recours dans des cas où la loi ne le prévoyait pas (CE, 21 janv. 1829, Brière, Rec. Lebon. p. 14), voire, après une disposition législative confirmant l'absence de recours. Et la jurisprudence, devenue célèbre, est venue confirmer ces premières décisions, toujours fondée sur la loi des 14 et 17 octobre 1790 , le droit d'agir en justice entrant dans la catégorie des garanties fondamentales accordées aux citoyens pour l'exercice des libertés publiques: CE, ass., 7 févr. 1947, d'Aillières, Rec. Lebon, p. 50 ; CE, ass., 17 févr. 1950, Ministre de l'Agriculture c/Dame Lamotte, Rec. Lebon, p. 110. Plus récemment le Conseil d'État est allé plus loin en se fondant sur le droit constitutionnellement reconnu à un recours juridictionnel effectif. Dès l'arrêt du 29 juillet 1998, Syndicat des avocats de France (Rec. Lebon, p. 313), la haute juridiction, visant la Constitution, indiquait qu'une atteinte au droit d'exercer un recours juridictionnel pouvait constituer la violation d'un principe à valeur constitutionnelle. Le Conseil d'État a été ensuite plus explicite en mentionnant «le principe à valeur constitutionnelle » du droit d'exercer un recours juridictionnel (CE, 11 déc. 2000, Bourahli, req. $\mathrm{n}^{\circ}$ 218812), le juge administratif paraissant aller même au-delà de la jurisprudence du Conseil constitutionnel en liant le droit à un recours au droit à l'aide juridictionnelle en tant que l'un peut rendre l'autre effectif (CE, 28 juill. 2000, $M E A$, req. $\mathrm{n}^{\circ} 151068$ ). Puis il a accueilli la jurisprudence du Conseil constitutionnel en indiquant que c'est l'article 16 de la DDHC qui fonde le droit à un recours juridictionnel effectif, tout en utilisant la formulation européenne pour préciser que ce droit ne saurait être atteint " dans sa substance même» (CE, 21 déc. 2001, M. et $M^{m e}$ Hoffmann, req. $\mathrm{n}^{\circ}$ 22862).

26. La Cour de cassation (ass. plén., 30 juin 1995, D. 1997, J., p. 515) a consacré l'accès effectif à un juge comme principe constitutionnel en le liant à celui du respect des droits de la défense, plus précisément à celui du droit à l'assistance d'un avocat: son «exercice effectif exige que soit assuré l'accès de chacun, avec l'assistance d'un défenseur, au juge chargé de statuer sur sa prétention». Puis, par un arrêt du 7 avril 2006 (bull. civ. 2006, ass. plén., $\mathrm{n}^{\mathrm{o}}$ 3), elle a repris la formulation de la Cour 
Ainsi, le droit d'accès à un tribunal apparaît désormais avec ce même caractère fondamental ${ }^{27}$, quoique sous des vocables différents, en droit européen des droits de l'homme, en droit communautaire, ainsi qu'en droit constitutionnel.

Après avoir posé le principe du droit d'accès, la Cour a ensuite développé une jurisprudence protectrice de l'individu, selon laquelle l'État est débiteur non seulement d'une obligation négative de s'abstenir d'entraver son droit d'accès à un tribunal, mais aussi, et au-delà, d'une obligation positive de lui en faciliter l'exercice ${ }^{28}$.

Cependant, la Cour européenne reconnaît aux États une certaine marge d'appréciation dans l'organisation de l'accès au juge. Le droit d'accès à un tribunal n'est pas absolu et peut faire l'objet de «limitations implicites ${ }^{29}$ ", sous réserve que celles-ci ne portent pas atteinte à la «substance même» du droit protégé et soient proportionnées au but légitime poursuivi ${ }^{30}$. D’où la possibilité de dégager de la jurisprudence de la Cour les exigences qu'elle a posées et les limitations qu'elle a validées.

Dès lors, au vu de ces affirmations renouvelées et renforcées du droit d'accès à un tribunal, éminemment jurisprudentielles et évolutives, et

européenne en énonçant «que si l'article $6 \$ 1$ de la CEDH permet à l'État de limiter le droit d'accès à un tribunal dans un but légitime, c'est à la condition que la substance même de ce droit n'en soit pas atteinte et que, si tel est le cas, les moyens employés soient proportionnés à ce but ».

27. Sur la notion de droits fondamentaux : voir Serge Guinchard, Droit processuel. Droit fondamentaux $d u$ procès, op. cit., $\mathrm{n}^{\circ} 44, \mathrm{p}$. 99, qui adopte la conception organique de Louis Favoreu, fondée sur trois critères: protection non seulement contre le pouvoir exécutif mais aussi législatif, contrairement aux libertés publiques en droit français; protection non seulement en vertu de la loi mais aussi et surtout en vertu de la Constitution et des textes internationaux ou supranationaux; protection non seulement par l'intervention des juges ordinaires mais aussi par celle d'un juge constitutionnel et de juges internationaux.

28. Renée Koering-Joulin, «Note relative au droit d'accès à un tribunal et à la chose jugée », en annexe au rapport du conseiller rapporteur, sous l'arrêt de l'ass. plén. du 7 juillet 2006 (Cesareo), pourvoi $n^{\circ}$ 04-10.672.

29. CEDH, 21 fév. 1975, Golder $c / R$.-U., préc., $\$ 38$.

30. CEDH, 28 mai 1985, Ashingdane $c / R$. $U$., série A 93, $\$ 57:$ : Le droit d'accès aux tribunaux n'est pas absolu; il peut donner lieu à des limitations implicitement admises car il appelle, de par sa nature même, une réglementation par l'État (qui peut varier dans le temps et dans l'espace en fonction des besoins et des ressources de la collectivité et des particuliers), laquelle ne doit en aucun cas porter atteinte à la substance dudit droit ni se heurter à d'autres droits consacrés par la Convention »; «Une limitation du droit d'accès ne serait compatible avec l'article 6 qu'à la double condition de poursuivre un but légitime et de présenter un rapport raisonnable de proportionnalité entre les moyens employés et le but visé. » 
afin de mieux en apprécier l'effectivité, il est utile de s'interroger, d'une part, sur les contours actuels de ces exigences (1) et, d'autre part, sur les limitations permises (2), au regard, le cas échéant, de leurs échos les plus saillants devant d'autres instances.

Il est particulièrement intéressant, en effet, de se demander, dans une perspective comparative, si la consécration supranationale ou constitutionnelle (en tout cas supra-législative) du droit d'accès au juge est le symbole de garanties plus étendues pour les justiciables.

\section{Les contours actuels des exigences découlant du droit d'accès à un tribunal}

\subsection{La notion de tribunal : un tribunal de pleine juridiction}

Tout d'abord, un «tribunal» au sens de la convention n'est pas nécessairement une juridiction au sens classique ou organique du terme et reçoit une acception autonome ${ }^{31}$.

C’est une définition matérielle et fonctionnelle qui doit prévaloir, caractérisée par la fonction juridictionnelle de l'instance considérée, à savoir «trancher, sur la base de normes de droit et à l'issue d'une procédure organisée, toute question relevant de sa compétence ${ }^{32} »$.

Cela suppose selon la terminologie de la Cour « un organe judiciaire de pleine juridiction ", c'est-à-dire que le contrôle du juge ne doit pas être trop limité sous peine de vider le droit au tribunal de sa substance ${ }^{33}$. En particulier, un contrôle restreint à l'examen de la motivation des faits et au détournement de la procédure ne suffit $\mathrm{pas}^{34}$. Il importe que soit exercé un contrôle complet de légalité et que le juge national soit compétent "pour les points de fait comme pour les questions de droit $^{35}$ ».

Ainsi, la compétence de décider, et plus précisément le pouvoir de rendre « une décision obligatoire, qu'une autorité non judiciaire n'aurait pas le pouvoir de modifier » est inhérente à la notion même de tribunal ${ }^{36}$.

31. CEDH, 27 août 2002, Didier c/. France, RDP 2003, p. 697, obs. Gérard Gonzalez: la Cour qualifie le conseil des marchés financiers de tribunal et s'assure que la procédure suivie devant lui remplit les exigences de l'article 6.

32. CEDH, 22 oct. 1984, Sramek c/ Autriche, série A 84, \$36, JDI 1985, 1070, obs. Paul Tavernier, à propos de l'autorité régionale des transactions immobilières du Tyrol.

33. CEDH, 10 fév. 1983, Albert et Le Compte c/ Belgique, série A 58, \$ 29.

34. CEDH, 28 juin 1990, Obermeier c/Autriche, série A 179, $\$ 70$.

35. CEDH, 21 sept. 1993, Zumtobel c/Autriche, série A 268.

36. CEDH, 19 avr. 1994, Van den Hurk c/Pays-Bas, série A 288, $\$ 45$. 
C'est sur ces bases que la Cour européenne a proscrit le mécanisme de renvoi préjudiciel du juge administratif français au ministre des Affaires étrangères, pour interprétation des traités internationaux ${ }^{37}$ ou pour l'appréciation de la condition de réciprocité dans leur application, inscrite à l'article 55 de la Constitution ${ }^{38}$.

Anticipant ${ }^{39}$ ou s'inspirant ${ }^{40}$ de ces arrêts, le Conseil d'État puis la Cour de cassation se sont reconnus compétents pour interpréter les traités internationaux.

Cette exigence du contrôle de "pleine juridiction" a conduit le Conseil d'État à exercer dans le cadre du recours pour excès de pouvoir un plein contrôle de proportionnalité sur les sanctions professionnelles, qui relèvent de la matière civile au sens de l'article 6, prononcées par une autorité administrative ${ }^{41}$.

Par ailleurs, en matière pénale, le recours de "pleine juridiction" suppose, de surcroît, que le juge détienne «le pouvoir de réformer en tous points, en fait comme en droit, la décision entreprise ${ }^{42} »$.

Toutefois, la Cour européenne valide le système français du retrait de point du permis de conduire, alors que le juge pénal n'exerce un contrôle que sur la culpabilité et non sur l'ampleur de la pénalité du retrait de points et qu'il ne peut individualiser la peine ${ }^{43}$.

Le Conseil d'État ${ }^{44}$ et le Conseil constitutionnel ${ }^{45}$ ont repris à leur compte cette analyse.

Il est donc permis de dégager de cette jurisprudence européenne l'exigence que le juge interne détienne la plénitude de juridiction avec un pouvoir de réformation, incluant un pouvoir de substitution pour les sanctions relevant de la matière pénale, à l'exclusion cependant des sanctions pour lesquelles le législateur a déjà prévu une modulation par la loi elle-même ${ }^{46}$.

37. CEDH, 24 nov. 1994, Beaumartin c/France, D. 1995, J, p. 235, note Xavier Prétot: le $\mathrm{CE}$ en abdiquant en la circonstance sa mission de juger et n'étant pas indépendant à l'égard du pouvoir exécutif, ne saurait mériter l'appellation de tribunal.

38. CEDH, 13 févr. 2003, Chevrol c/France, D. 2003, J., note Hugues Moutouh.

39. CE, ass., 29 juin 1990, Gisti, Rec. Lebon, p. 171.

40. Cass. civ., 19 déc. 1995, Banque africaine de développement, RGDIP 1996, p. 867, obs. Denis Alland.

41. CE, 22 juin 2007, M. Arfi, RFDA 2007, p. 1199, concl. Mattias Guyomar.

42. CEDH, 23 oct. 1995, Schmautzer et autres c/Autriche, série A 328.

43. CEDH, 23 sept. 1998, Malige c/France, JCP G 1999, II, 10086, note Frédéric Sudre.

44. CE, avis, 27 sept. 1999, Rouxel, $\mathrm{n}^{\mathrm{O}} 208242$.

45. Déc. $\mathrm{n}^{\circ} 99-411$ DC, 16 juin 1999.

46. Frédéric Sudre, Droit européen et international des droits de l'homme, op. cit., p. 375. 
À cet égard, à la différence du Conseil d'État ${ }^{47}$ et de la Cour de Strasbourg ${ }^{48}$ qui estiment que les dispositions de l'article 6 n'impliquent pas que le juge de l'impôt puisse moduler les pénalités fiscales en fonction de la gravité de la faute commise par le contribuable, la Cour de cassation ${ }^{49}$ affirme qu'un « recours de pleine juridiction » doit permettre « au tribunal de se prononcer sur le principe et le montant de l'amende». Le juge européen a toutefois confirmé sa position récemment ${ }^{50}$.

Enfin, la Cour européenne admet depuis longtemps que des « impératifs de souplesse et d'efficacité » puissent justifier l'intervention dans la procédure en première instance, en matière civile ou pénale, d'organes non juridictionnels, administratifs ou disciplinaires, qui ne soient pas intégrés aux « structures judiciaires ordinaires » et qui ne satisfassent pas aux garanties de l'article $6^{51}$, à la condition que le justiciable dispose d'un recours devant un organe judiciaire indépendant, doté de la plénitude de juridiction et offrant les garanties de l'article $6^{52}$ : le droit à un tribunal est ainsi susceptible d'être différé dans le temps.

\subsection{L'accès à un tribunal s'entend en droit européen d'un accès concret et effectif au juge ${ }^{53}$}

Cette exigence présume que le justiciable «jouisse d'une possibilité claire et concrète de contester un acte constituant une ingérence dans ses

47. CE, 17 déc. 2003, Nguyen, Rec. Lebon. Tab., $\mathrm{n}^{0} 247988$.

48. CEDH, 27 janv. 2005, Fattell c/France, AJDA 2005, p. 1388, note Delphine Costa.

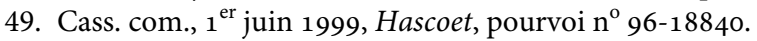

50. CEDH, 7 juin 2012, Segame SA c/France, Dr. fisc. 2012, commentaire de Ludovic Ayrault : arguant $(\$ 56)$ de la spécificité du contentieux fiscal qui, d'une part, implique une exigence d'efficacité nécessaire pour préserver les intérêts de l'État, et, d'autre part, «ne fait pas partie du noyau dur du droit pénal au sens de la Convention », la Cour juge que le fait que les juridictions administratives n'aient pas le pouvoir de moduler une amende fiscale (CGI, ex-art. 1770 octies) ne contrevient pas à l'exigence du contrôle de la sanction par un tribunal de «pleine juridiction » dès lors que, dans le cadre d'un recours de plein contentieux, le juge administratif «apprécie tous les éléments de fait et de droit et peut non seulement annuler ou valider un acte administratif mais également le réformer, voire substituer sa propre décision à celle de l'administration et se prononcer sur les droits de l'intéressé » : ce faisant la Cour valide le système français des pénalités fiscales et leurs modalités de contrôle par le juge national.

51. CEDH, 23 juin 1981, Le Compte, Van Leuwen et De Meyere c/Belgique, req. $\mathrm{n}^{\circ} 6878 / 75$ et $7238 / 75$.

52. CEDH, 10 févr. 1983 , Albert et Le Compte c/Belgique, op. cit., $\$ 29$.

53. $\mathrm{CEDH}, 9$ oct. 1979, Airey c/Irlande, série $\mathrm{A} \mathrm{n}^{\circ}$ 32, dans lequel la Cour énonce que «la Convention a pour but de protéger des droits, non pas théoriques et illusoires, mais concrets et effectifs»; voir l'article de Michel Hottelier, en ligne à l'adresse: http://www.Rtdh.eu/pdf/2007573.pdf. 
droits $^{54}$ ». Autrement dit, afin d'assurer l'effectivité de l'accès au tribunal, les États ont l'obligation positive de lever la plupart des obstacles qui peuvent entraver cet accès.

Par ailleurs, le caractère effectif doit s'apprécier au cas par cas, en tenant compte des faits de l'espèce, méthode qui laisse une grande marge de manœuvre au juge européen ${ }^{55}$.

Les obstacles donnant lieu à la censure de la Cour peuvent être financiers (1.2.1), juridiques (1.2.2) ou de fait (1.2.3).

\subsubsection{Les obstacles financiers}

Il peut s'agir d'une consignation trop élevée ou bien de l'impossibilité d'obtenir une aide judiciaire dans une procédure qui la justifie.

Dans cette dernière hypothèse, si le droit à l'aide judiciaire gratuite n'est expressément garanti qu'en matière pénale (article $6 \$ 3$ ), la Cour européenne considère que méconnaît le droit à un tribunal, l'impossibilité d'obtenir l'assistance judiciaire gratuite dans le cadre d'une procédure "civile» lorsque la complexité de la procédure ou de la cause rend cette assistance indispensable ${ }^{56}$ ou que la loi prescrit la représentation d'un avocat ${ }^{57}$, ce d'autant que le défaut d'aide judiciaire peut entraîner une rupture de l'égalité des armes ${ }^{58}$, en particulier lorsque l'adversaire dispose de moyens matériels importants pour assurer sa défense ${ }^{59}$.

La Cour semble cependant avoir atténué la rigueur de sa jurisprudence sur l'aide juridictionnelle en admettant, tout d'abord, dans une procédure sans représentation obligatoire ${ }^{60}$ que le refus d'accorder celleci pour défaut d'un moyen sérieux de cassation était compatible avec

54. CEDH, 4 déc. 1995, Bellet c/France, $\$ 36$, D. 1997, p. 205, note Sophie Perez.

55. CEDH, 20 nov. 1995, British American Tobacco Co. Ltd. c/Pays-Bas, req. $\mathrm{n}^{\mathrm{O}} 19589 / 92$.

56. CEDH, 9 oct. 1979, Airey c/Irlande, op. cit.

57. CEDH, 30 juil. 1998, Aerts c/Belgique, $\mathrm{n}^{\circ}$ 25357/94 : refus d'accorder l'aide juridictionnelle dans une procédure avec représentation obligatoire, au motif que la prétention ne paraissait pas sérieuse.

58. CEDH, ${ }_{3}$ févr. 2003, Bertuzzi c/France, $n^{0}$ 36378/97 : l'aide juridictionnelle avait été accordée mais les avocats successifs se sont désistés en raison de leur lien personnel avec l'avocat attaqué, le requérant s'est trouvé seul à défendre sa cause contre un professionnel du droit.

59. CEDH, 15 févr. 2005, Steel et Morris c/R.-U., $\mathrm{n}^{0}$ 68416/01; AJDA 2005, p. 1895, chron. Jean-François Flauss.

60. CEDH, 19 sept. 2000, Gnahore c/France, RDP 2001, p. 682, obs. Adeline Gouttenoire. 
l'article 6, dès lors que «le système mis en place par le législateur offre des garanties substantielles aux individus, de nature à les préserver de l'arbitraire ", tenant d'une part à la composition du bureau d'aide juridictionnelle et, d'autre part, à la possibilité d'un recours contre ses décisions de rejet. Elle a ensuite opéré la même analyse dans une procédure avec représentation obligatoire ${ }^{61}$.

Dans une perspective semblable elle juge que l'obligation de constituer avocat devant une cour suprême n'est pas contraire au droit d'accès à un tribunal $^{62}$.

De même, l'article 575 du Code de procédure pénale français, qui, hors les cas limitativement énumérés, subordonne le droit pour la partie civile de former un pourvoi en cassation contre un arrêt de non-lieu de la chambre d'instruction, à l'exercice de cette voie de recours par le ministère public, ne porte pas atteinte à la substance même du droit du justiciable d'accéder à un tribunal ${ }^{63}$.

En second lieu, le droit d'accès à un tribunal peut être méconnu par le montant excessif d'une consignation fixé pour une plainte avec constitution de partie civile ${ }^{64}$ ou par des frais de procédure trop élevés ${ }^{65}$ au regard de la capacité financière du justiciable.

\subsubsection{Obstacles juridiques}

La complexité de la procédure peut constituer un obstacle méconnaissant le droit d'accès au juge ${ }^{66}$. Il en est de même de l'incapacité d'ester en justice du fait de la législation de l'État ${ }^{67}$ ou encore, de l'absence en droit national d'un accès direct à un tribunal pour introduire une demande en rétablissement de capacité : la Cour dégage à la fois, de la «tendance » existant au niveau européen et des instruments internationaux de protection des personnes atteintes de troubles mentaux, une « orientation " qui la conduit à interpréter l'article 6 comme «garantissant en principe

61. CEDH, 26 févr. 2002, Del Sol c/France, RDP 2003, p. 3, note Adrien Gouttenoire.

62. CEDH, 24 nov. 1986, Gillow c/R.-U., série $\mathrm{A} \mathrm{n}^{\circ}$ 109, $\$ 69$; CEDH, 26 juill. 2002, Meftah c/France, D. 2003, p. 594, obs. Nathalie Fricero concernant les avocats aux conseils.

63. CEDH, 3 déc. 2002, Berger c/France, req. $\mathrm{n}^{\mathrm{o}}$ 48221/99, RDP 2003, p. 3, obs. Christine Hugon.

64. CEDH, 28 oct. 1998, Aït-Mouloub c/France, req. $\mathrm{n}^{0} 22924 / 93$.

65. CEDH, 19 juin 2001, Kreuz c/Pologne, req. $\mathrm{n}^{\circ} 28249 / 95$.

66. CEDH, 16 déc. 1992, De Geouffre de La Pradelle c/France, série A n ${ }^{\circ}$ 253-B, \$ 37 : à propos des modalités d'exercice d'un recours devant le Conseil d'État en annulation d'un décret de classement d'un site.

67. CEDH, 16 déc. 1997, Église catholique de la Canée c/Grèce, req. n $^{\circ}$ 25528/94. 
à toute personne déclarée partiellement incapable [...] un accès direct à un tribunal pour demander le rétablissement de sa capacité juridique ${ }^{68}$ " $(\$ 245)$.

Le Conseil constitutionnel français sanctionne également dans sa jurisprudence récente les obstacles juridiques privant les intéressés d'un recours effectif devant une juridiction.

Par exemple, dans sa décision $\mathrm{n}^{\circ}$ 2013-314 QPC du 14 juin 2013, prise après la saisine, pour la première fois de la CJUE d'une question préjudicielle ${ }^{69}$, le Conseil a jugé - après l'arrêt de la Cour de Luxembourg du 30 mai 2013 précisant l'interprétation ${ }^{70}$ de la décision-cadre du 13 juin 2002 relative au mandat d'arrêt européen -, qu'en privant les parties de la possibilité de former un recours en cassation contre l'arrêt de la chambre de l'instruction statuant sur une demande, soit d'extension des effets du mandat à d'autres infractions, soit d'autorisation de la remise de la personne à un État tiers, les dispositions contestées de l'article 695-46 du Code de procédure pénale apportent une restriction injustifiée au droit à exercer un recours juridictionnel effectif. Par suite, le Conseil a jugé les mots « sans recours ", figurant au quatrième alinéa de cet article, contraires à la Constitution.

De même, dans sa décision n ${ }^{0}$ 2014-390 QPC du 11 avril 2014, M. Antoine $H$., le Conseil a jugé, qu'en permettant au procureur de la République d'ordonner la destruction de biens saisis sans que, notamment, le propriétaire intéressé ait été mis à même de contester cette décision devant une juridiction afin de demander, le cas échéant, leur restitution, le quatrième alinéa de l'article 41-4 du Code de procédure pénale méconnait le droit à un recours effectif devant une juridiction.

68. CEDH, 17 janv. 2012, Stanev c/Bulgarie, req. $\mathrm{n}^{\mathrm{o}} 36760 / 06$; Voir Béatrice PastreBelda, JCP G 2012, act. 142 ; la Cour fait valoir, en premier lieu, que le droit de demander à un tribunal de réviser une déclaration d'incapacité "constitue l'un des droits procéduraux essentiels pour la protection des personnes déclarées partiellement incapables », car « une telle procédure est déterminante pour l'exercice de l’ensemble des droits et libertés affectés par la déclaration d'incapacité» (\$242); si, en second lieu, il est établi que le droit d'accès à un tribunal n'est pas absolu et que l'État demeure libre de déterminer les modalités procédurales pour l'exercice de cet accès direct, encore faut-il que soient mis en œuvre des «moyens moins restrictifs qu'une privation automatique de l'accès direct ».

69. Décision $\mathrm{n}^{\mathrm{o}}$ 2013-314 QPC du 4 avril 2013.

70. CJUE, 30 mai 2013, Jeremy F., C-168/13 PPU, ECLI : EU : C: 2013: 358, qui y énonce le fait que cette décision-cadre ne s'oppose pas à ce que les États membres prévoient un recours suspendant l'exécution de la décision de l'autorité judiciaire qui statue dans un délai de 30 jours à compter de la réception de la demande. 
Ou encore, en matière de procédure devant la cour d'assises, dans sa décision $\mathrm{n}^{\mathrm{0}}$ 2014-403 QPC du 13 juin 2014, M. Laurent L., le Conseil a jugé que les dispositions du cinquième alinéa de l'article 380-11 du Code de procédure pénale, qui prévoient qu'en cas d'appel, lorsque l'accusé a pris la fuite et n'a pu être retrouvé avant l'ouverture de l'audience ou au cours de son déroulement, le président de la cour d'assises constate la caducité de l'appel, portent une atteinte disproportionnée au droit à un recours juridictionnel effectif.

\subsubsection{Les obstacles de fait}

L'accès effectif au juge peut aussi être méconnu par des carences diverses des institutions, comme celle des autorités pénitentiaires à transmettre dans les délais au greffe de la cour d'appel l'appel du détenu ${ }^{71}$ ou encore par le délai d'examen d'un pourvoi le rendant sans objet ${ }^{72}$.

\subsection{Le droit d'accès à un tribunal emporte celui d'obtenir une décision de justice motivée et tranchant définitivement le litige}

\subsubsection{Une décision motivée}

Le droit d'accès implique le droit de présenter ses arguments et celui d'être vraiment «entendu» : le tribunal doit donc procéder à un examen effectif des moyens de parties et à une motivation appropriée du jugement. Un moyen susceptible d'une incidence décisive pour la solution requiert une réponse spécifique et explicite ${ }^{73}$, par exemple un moyen ayant trait aux droits garantis par la convention ${ }^{74}$.

Cependant, s'agissant de la procédure d'admission des pourvois en cassation (loi du 25 juin 2001) la Cour a considéré que l'article $6 \$ 1$ n'exige pas que soit motivée en détail une décision par laquelle une juridiction de recours, se fondant sur une disposition légale spécifique, écarte un recours comme dépourvu de chance de succès ${ }^{75}$.

71. CEDH, 17 janv. 2006, Barbier c/France, req. $\mathrm{n}^{0}$ 76093/01, D. 2006, p. 1209, note Fabrice Defferard et Vincent Durtette.

72. CEDH, 26 juil. 2007, Schmidt c/France, $\mathrm{n}^{\circ}$ 35109/02, JCP G 2008, I, 102, obs. Adeline Gouttenoire.

73. $\mathrm{CEDH}, 9$ déc. 1994, Ruiz Torija et Hiro Balani c/Espagne, série $\mathrm{A} \mathrm{n}^{\circ}$ 303-A et $\mathrm{A}$ $\mathrm{n}^{\mathrm{o}} 303-\mathrm{B}$; voir CEDH, 19 févr. 1998, Higgins et autres c/France, req. $\mathrm{n}^{\mathrm{o}} 20124 / 9$.

74. CEDH, 28 juin 2007, Wagner et J.M.W.L. c/Luxembourg, req. $\mathrm{n}^{\circ} 76240 / 01$.

75. CEDH, 28 janv. 2003, Burg c/France, $n^{\circ} 34763 / 02$ : à propos de la procédure d'admission des pourvois. 


\subsubsection{Une décision tranchant définitivement le litige}

Le droit d'accès à un tribunal emporte le respect du principe de la sécurité juridique, qui participe de la prééminence du droit et qui présume que «la solution donnée de manière définitive à tout litige par les tribunaux ne soit plus remise en cause ${ }^{76} »$.

Par exemple, c'est en se fondant sur l'article $6 \$ 1$ que la Cour de cassation française limite la portée d'un revirement de jurisprudence et juge que cette disposition s'oppose à l'application immédiate à un demandeur en justice de l'interprétation nouvelle d'une règle de prescription qui aurait pour effet de lui interdire l'accès au juge $\mathrm{e}^{77}$.

Surtout, la Cour européenne a opéré à ce titre un contrôle des lois de validation rétroactives, qu'elle a progressivement étendu aux lois expressément rétroactives ${ }^{78}$ ainsi qu'aux lois interprétatives ${ }^{79}$.

Toutefois, le choix du fondement juridique retenu par la Cour européenne pour opérer ce contrôle a fait l'objet d'importantes variations: droit d'accès à un tribunal ${ }^{80}$, égalité des armes ${ }^{81}$ ou équité de la procédure en général ${ }^{82}$.

L'évolution de sa jurisprudence a abouti au principe selon lequel le droit à un procès équitable ne prohibe pas de manière absolue l'intervention du législateur dans le cours d'une procédure juridictionnelle pour en modifier l'issue. En effet, après avoir paru condamner le principe même des interventions législatives ${ }^{83}$, la Cour a nuancé sa position en jugeant que «l'article $6 \$ 1$ ne saurait s'interpréter comme empêchant toute

76. CEDH, 28 oct. 1999, Brumarescu c/Roumanie, req. $\mathrm{n}^{\circ} 28342 / 95$.

77. Cass., ass. plén., 21 déc. 2006, Sté La Provence $c / M^{m e} X$, pourvoi $n^{0}$ oo-2049.

78. CEDH, 6 oct. 2005, Draon c/France et Maurice c/France, req. $\mathrm{n}^{\circ} 1513 / 03$ : application immédiate de la loi du 4 mars 2002, dite loi anti-Perruche, aux instances en cours.

79. CEDH, 14 févr. 2006, Lecarpentier c/France, req. $\mathrm{n}^{\circ} 67848 / 01$, D. 2006, p. 717 , obs. Céline Rondey.

80. CEDH, 20 févr. 2003, Forrer-Niedenthal c/Allemagne, req. $\mathrm{n}^{\circ} 47316 / 99$.

81. CEDH, 9 déc. 1994, Raffineries grecques Stran et Stratis Andreadis c/Grèce, série A $\mathrm{n}^{\circ}$ 301-B, $\$ 49$; CEDH, 27 mai 2004, Ogis-Institut Stanislas et autres c/France, req. $\mathrm{n}^{\mathrm{o}} 42219 / 98$ et $54563 /$ oo.

82. CEDH, 28 oct. 1999, Zielinski, Pradal, Gonzalez et al c/France, req. $n^{0} 34173 / 96$.

83. CEDH, 9 déc. 1994, Raffineries grecques Stran et Stratis Andreadis c/Grèce, op. cit.: au motif que «le principe de la prééminence du droit et la notion de procès équitable consacrés par l'article 6 s'opposent à toute ingérence du pouvoir législatif dans l'administration de la justice dans le but d'influer sur le dénouement judiciaire d'un litige». 
ingérence des pouvoirs publics dans une procédure judiciaire pendante à laquelle ils sont parties ${ }^{84}$ ".

Et la Cour a dégagé trois conditions auxquelles est subordonnée la conformité de la loi de validation: le caractère non définitif de la procédure juridictionnelle en cause, la proportionnalité de l'atteinte au droit d'accès à un tribunal (au but légitime) et l'existence d'un «motif impérieux d'intérêt général ».

L'appréciation stricte de l'existence d'un motif impérieux d'intérêt général a conduit la Cour européenne à juger ${ }^{85}$ qu'une loi de validation déclarée conforme à la Constitution par la Conseil constitutionnel n'est pas nécessairement compatible avec l'article $6 \$ 1$.

À cet égard, il ressort de la jurisprudence de la Cour que le motif de sauvegarder l'équilibre financier de tel ou tel secteur économique, souvent évoqué par les pouvoirs publics, ne justifie pas pour elle, en principe, à lui seul, l'intervention du législateur ${ }^{86}$.

Par ailleurs, une loi rétroactive étant également susceptible de porter atteinte au droit de propriété, le contrôle de l'existence ou non d'un motif impérieux d'intérêt général est opéré indistinctement par la Cour au regard de l'article $6 \$ 1$ et/ou de l'article 1 du protocole $1^{87}$.

Le Conseil constitutionnel français a également fait du respect des décisions de justice définitives et de la présence d'un motif d'intérêt général, des conditions de la conformité d'une loi de validation à la Constitution $^{88}$, tandis que l'arrêt Zielinski l'a conduit à renforcer ces conditions et à durcir son contrôle ${ }^{89}$.

Très récemment, par une décision $\mathrm{n}^{\mathrm{0}}$ 2013-366 QPC du 14 février 2014, le Conseil constitutionnel a "parachevé l'évolution de sa jurisprudence sur les validations législatives, initiée il y a quinze ans ${ }^{90}$ ",

84. CEDH, 23 oct. 1997, National et Provincial Building Society et al. c/Royaume-Uni, req. $\mathrm{n}^{\mathrm{o}} 21319 / 93, \mathrm{n}^{\circ} 21449 / 93$ et $\mathrm{n}^{\circ} 21675 / 93, \$ 112$ : la loi validait rétroactivement des dispositions fiscales objet de procédures pendantes et orientait en faveur de l'administration fiscale des instances auxquelles celle-ci était partie.

85. CEDH, 28 oct. 1999, Zielinski, Pradal, Gonzalez et autres c/France, op. cit.

86. CEDH, 9 janv. 2007, Arnolin et autres c/France, $\mathrm{n}^{\circ}$ 20127/03; CEDH, 11 avr. 2006, Cabourdin c/France, $\mathrm{n}^{\circ}$ 60796/o0, JCP G 2006, I, 164, chron. Frédéric Sudre.

87. CEDH, Draon c/France et Maurice c/France, op. cit.

88. Conseil const., 19 nov. $1997, \mathrm{n}^{\circ}$ 97-328 DC.

89. Conseil const., 21 déc. 1999, $\mathrm{n}^{\circ}$ 99-422 DC; Cons. const., 29 déc. 1999, $n^{\circ} 99-425$ DC.

90. Voir le communiqué de presse du Conseil, en ligne sur http://www.conseilconstitutionnel.fr, qui précise que « ce faisant, le Conseil constitutionnel a entendu expressément souligner l'exigence de son contrôle : le contrôle des lois de validation qu'il assure sur le fondement de l'article 16 de la Déclaration de 1789 a la même 
en remplaçant, dans son considérant de principe sur le contrôle des lois de validation, la référence à un "intérêt général suffisant», par la référence à «motif impérieux d'intérêt général ». Et selon le Conseil luimême: «Désormais, les exigences constitutionnelles et conventionnelles se rejoignent entièrement ${ }^{91}{ }$.

La Cour de cassation ${ }^{92}$ comme le Conseil d'État ${ }^{93}$ ont aussi progressivement évolué vers un ralliement à la notion d'«impérieux motifs d'intérêt général » pour contrôler les lois rétroactives.

Enfin, la Cour européenne a finalement décidé que le contrôle des lois rétroactives «va au-delà des litiges dans lesquels l'État est partie ${ }^{94}$ » et s'étend aux litiges de droit privé entre particuliers.

portée que le contrôle assuré sur le fondement des exigences qui résultent de la CESDH » et que «le Conseil a, en l'espèce, jugé conforme à la Constitution l'article 50 de la loi du 29 décembre 2012 de finances rectificative pour 2012 qui valide les délibérations des syndicats mixtes instituant le "versement transport" adoptées avant le $1^{\mathrm{er}}$ janvier 2008 en ce que leur légalité serait contestée par le moyen tiré de l'incompétence de ces syndicats pour établir cette imposition ". S'agissant de l'existence d'un motif impérieux d'intérêt général, le Conseil a ainsi considéré que "le législateur a entendu mettre un terme à des années de contentieux relatives aux délibérations des syndicats mixtes instituant le "versement transport】» et qu' "il a également entendu éviter une multiplication des réclamations fondées sur la malfaçon législative révélée par les arrêts précités de la Cour de cassation et tendant au remboursement d'impositions déjà versées et mettre fin au désordre qui s'en est suivi dans la gestion des organismes en cause» (cons. 6). Le motif impérieux d'intérêt général réside donc essentiellement dans la volonté du législateur de mettre fin à une incertitude juridique, source d'un abondant contentieux, et à éviter les nombreuses réclamations résultant de la reconnaissance par la Cour de cassation de l'incompétence des syndicats mixtes pour ordonner le « versement transport » avant l'adoption de la loi du 24 décembre 2007. Le Conseil a également reconnu que les dispositions contestées réservaient expressément les décisions passées en force de chose jugée. Il a, enfin, veillé à ce que la loi de validation respecte le principe de non-rétroactivité des peines et des sanctions, garanti par l'article 8 de la DDHC. Il a donc formulé une réserve pour que la validation rétroactive des délibérations instituant le "versement transport» ne puisse donner lieu à des sanctions qui pourraient être prononcées à l'encontre des contribuables ne s'étant pas acquittés de cette imposition.

91. Voir le communiqué de presse du Conseil constitutionnel déjà cité.

92. Cass. civ. $1^{\text {re }}, 9$ juill. 2003, Epx Dolléans c/SA Union de crédit pour le bâtiment, pourvoi $\mathrm{n}^{\circ}$ 99-15213: concernant l'« équilibre financier de l'ensemble des établissements de crédit».

93. CE, 23 juin 2004, Société "Laboratoire Génevrier", $\mathrm{n}^{\circ}$ 257797, s'agissant de «l'équilibre financier du système de santé national».

94. CEDH, 9 janv. 2007, Arnolin et autres c/France, op. cit.; CEDH, 11 avr. 2006, Cabourdin c/France, op. cit. 
De facto, le juge interne avait ouvert la voie et anticipé l'interprétation de la Cour européenne ${ }^{95}$.

\section{Les limitations au droit d'accès à un tribunal}

Le principe d'admettre des limitations au droit d'accès à un tribunal a été affirmé assez tôt par la Cour de Strasbourg, qui a ainsi décidé que ce droit n'était pas absolu et pouvait faire l'objet de «limitations implicites ${ }^{96} "$, sous réserve que celles-ci ne portent pas atteinte à la «substance même ${ }^{97}$ » du droit protégé et soient "proportionnées» au «but légitime» recherché.

La position de la Cour européenne diffère à cet égard de celle du Comité des droits de l'homme de l'ONU qui considère que "le droit d'être jugé par un tribunal indépendant et impartial est un droit absolu qui ne souffre aucune exception ».

La notion d'atteinte à la substance même du droit à un tribunal a été interprétée en droit interne par la Cour de cassation. Par exemple en matière d'action en garantie des vices cachés, la condition de «bref délai » prévue par l'article 1641 du Code civil ne porte pas atteinte à la substance même du droit d'accès à un tribunal ${ }^{98}$.

La haute juridiction estime en revanche que les dispositions adoptées en faveur du désendettement des rapatriés réinstallés dans une profession non salariée, qui prévoient une suspension automatique des poursuites sans l'intervention d'un juge et d'une durée indéterminée, portent atteinte à la substance même du droit des créanciers à un tribunal ${ }^{99}$.

De son côté, le juge européen a considéré que le principe de concentration des moyens posé par la Cour de cassation par arrêt de l'assemblée plénière du 7 juillet $2006^{100}$ ne porte pas atteinte à la substance même

95. Cass., ass. plén., 23 janv. 2004, SCI Le Bas Noyer c/Castorama, pourvoi $\mathrm{n}^{\mathrm{o}}$ 03-13617: qui énonce que la règle européenne gouvernant les validations législatives s'applique «même lorsque l'État n'est pas partie au litige » et « quelle que soit la qualification formelle donnée à la loi » et vaut donc pour une loi interprétative.

96. CEDH, 21 févr. 1975, Golder c/R.-U., op. cit., $\$ 38$.

97. CEDH, 28 mai 1985, Ashingdane c/R.-U., op. cit. ; CEDH, 11 janv. 2005, Musemeci $c /$ Italie, req. $\mathrm{n}^{\mathrm{o}} 33695 / 96$, censurant l'absence de recours contre une mesure pénitentiaire de "surveillance renforcée», analysée en droit interne comme une "manifestation du pouvoir discrétionnaire de l'administration dans le cadre de l'organisation de la vie au sein des établissements pénitentiaires ".

98. Cass. civ. $1^{\mathrm{re}}, 21$ mars 2000, Le Collinet $c / C^{i e}$ d'Assurance Rhin et Moselle, pourvoi $n^{\circ} 98-11982$.

99. Cass., ass. plén., 7 avr. 2006, SCI Barnabé c/SARL Building, pourvoi n ${ }^{\circ}$ 05-11519.

100. Cass., ass. plén., 7 juill. 2006, pourvoi n ${ }^{\circ}$ 04-10.672. 
du droit d'accès à un tribunal, en ce qu'il tend à assurer une bonne administration de la justice, à réduire le risque de manœuvres dilatoires et à favoriser une décision dans un délai raisonnable ${ }^{101}$.

De même, il estime que le refus de transmission d'une question prioritaire de constitutionnalité, au regard « des critères de non-renvoi » prévus par la loi, ne constitue pas une atteinte injustifiée au droit d'accès au Conseil constitutionnel.

Les limitations les plus saillantes au droit d'accès à un tribunal résultent d'immunités de juridictions (2.1), de règles procédurales de droit interne (2.2) ou encore de règles subordonnant l'accès au tribunal à l'exécution d'une décision antérieure (2.3).

\subsection{Les immunités de juridictions}

La Cour européenne n'écarte ces immunités que lorsqu'elle les analyse comme des obstacles procéduraux interdisant au requérant de porter une demande en réparation devant le juge national. Mais la référence au seul droit interne pour déterminer l'existence d'un «droit » à réparation au sens de l'article 6 conduit à une distinction, critiquée en doctrine ${ }^{102}$, entre les limitations matérielles d'un droit consacré par la législation interne, auxquelles l'article 6 ne s'applique pas, et les limitations procédurales, auxquelles l'article 6 s'applique au titre du droit d'accès à un tribunal.

Par exemple dans l'affaire Roche c/Royaume-Uni ${ }^{103}$, le requérant est dans l'impossibilité d'agir en réparation contre le ministère de la Défense en application de l'article 10 de la loi de 1947 relative aux procédures concernant la Couronne. Cette disposition légale interdit aux membres des forces armées blessés ou décédés dans l'exercice de leurs fonctions d'engager contre la Couronne une action en responsabilité civile lorsque le ministre atteste que le décès ou le dommage imputable est considéré comme imputable au service aux fins du droit à une pension de guerre.

101. CEDH, 17 mars 2015, Barras c/France, req. $\mathrm{n}^{\circ}$ 12686/10.

102. Frédéric Sudre, Droit européen et international des droits de l'homme, op. cit., $\mathrm{n}^{\mathrm{o}} 212-4$, p. 386.

103. CEDH, 19 oct. 2005, Roche c/R.-U., req. $\mathrm{n}^{0} 32555 / 96$, JCP G 2006, I, 109, chron. Frédéric Sudre ; $\mathrm{CEDH}, 14$ déc. 2006, Markovic et al. c/Italie, req. $\mathrm{n}^{\mathrm{o}}{ }_{1398 / 03} \$ 114$ : l'impossibilité de mettre en jeu la responsabilité de l'État pour un acte de gouvernement est analysée non comme une règle consacrant une immunité de juridiction mais comme une règle régissant «le droit d'action matériel en droit interne » qui, selon la Cour, ne méconnaît pas le droit d'accès à un tribunal alors même que les requérants ne peuvent pas obtenir une décision sur le bien-fondé de leur demande. 
Une telle limitation qui interdit au requérant de se prévaloir d'un quelconque droit à réparation n'est pas analysée comme une limitation procédurale et l'article 6 ne s'y applique donc pas.

Dans une perspective analogue, dans l'affaire Jones et autres c/RoyaumeUni du 14 janvier 2014, les tribunaux anglais ont accueilli la demande d'immunité présentée par l'Arabie Saoudite à l'encontre de l'action civile en réparation engagée par quatre ressortissants britanniques contre l'Arabie Saoudite et contre des fonctionnaires de cet État pour des actes de torture que ces derniers leur auraient infligés sur le territoire saoudien.

La Cour confirme la solution de son arrêt Al Adsani c/RoyaumeUni du 21 novembre 2001, en s'inclinant, comme le commente un auteur ${ }^{104}$, «devant une doctrine de l'immunité des États anachronique et incompatible avec le respect des droits de l'homme». La Cour répète que l'immunité de juridiction de l'État, qui poursuit «le but légitime» de respecter un principe de droit international protecteur de la souveraineté des États, ne peut «de façon générale» passer pour «disproportionnée» puisqu'elle reflète «des principes de droit international généralement reconnus en matière d'immunité des États » destinés à favoriser la courtoisie et les bonnes relations entre États ${ }^{105}$. Elle estime que l'immunité de l'État offre en principe aux agents de l'État, à raison des actes accomplis pour le compte de ce dernier, la même protection que celle accordée à l'État lui-même, ceci afin d'éviter que le droit pour l'État à l'immunité ne soit contourné en assignant à sa place certains de ses agents nommément désignés.

Dans la même veine, la Cour juge que l'immunité des organisations internationales constitue une «limitation implicite» du droit d'accès à un tribunal compatible avec l'article 6 dès lors que les requérants disposent en droit interne d'autres voies de droit pour protéger leurs droits garantis par la convention.

Cependant, le juge européen renonce à un tel contrôle de l'existence d'une voie de recours alternative concernant les immunités de juridiction des États.

La même analyse est suivie par la Cour en droit interne concernant les immunités des parlementaires, des fonctionnaires ou des magistrats,

104. Frédéric Sudre, Droit à un procès équitable, chronique, JCP G 14 juillet 2014, $\mathrm{n}^{\circ} 28$, doct. $832, n^{\circ} 4$.

105. CEDH, 21 nov. 2001, Al-Adsani c/R.-U., req. $\mathrm{n}^{\circ}$ 35763/97, Fogarty c/R.-U., req. $\mathrm{n}^{\mathrm{o}} 37112 / 97$ et Mc Elhinney c/Irlande, req. $\mathrm{n}^{\mathrm{o}} 31253 / 96$. 
la Cour vérifiant bien en ces cas l'existence de voie de recours de substitution offerte au particulier.

Toutefois, une immunité générale et absolue ayant pour objet de «soustraire à la compétence des tribunaux toute une série d'actions civiles ou d'exonérer de toute responsabilité civile de large groupe de personnes» est condamnée par la Cour en principe ${ }^{106}$.

Ainsi, les immunités de juridiction et d'exécution et, en particulier, celles dont bénéficient les États, sont désormais encadrées à la fois par une construction jurisprudentielle de droit interne fondée sur la coutume internationale et les règles du droit international public gouvernant les relations entre États, ainsi que par l'appréciation par le juge européen au cas par cas de leur compatibilité avec le droit d'accès à un tribunal.

Il convient de relever à cet égard que la jurisprudence française est passée au $\mathrm{xx}^{\mathrm{e}}$ siècle d'une conception générale et absolue de l'immunité de juridiction, à une conception plus restrictive fondée essentiellement sur la distinction entre les "actes d'autorité », couverts par les immunités, et les «actes de gestion» qui en sont exclus. Dans le même sens, la France a ratifié le 20 août 2011 la convention des Nations unies du 2 décembre 2004 sur les immunités juridictionnelles des États et de leurs biens, dont la Cour de cassation s'inspire déjà, bien qu'elle ne soit pas encore entrée en vigueur, et qui reprend cette distinction, tout en interdisant aux États de se prévaloir d'une immunité absolue.

\subsection{Les règles procédurales internes}

La Cour européenne sanctionne comme portant atteinte à la substance du droit d'accès à un juge les règles procédurales empêchant ou interdisant à certains sujets de droit, personnes physiques ou personnes morales, d'agir en justice ${ }^{107}$.

Mais elle admet en principe que des délais de prescription, justifiés par les nécessités de préserver la sécurité juridique, viennent limiter le droit d'accès à un tribunal ${ }^{108}$.

Toutefois, elle considère, au vu de circonstances exceptionnelles, que le droit d'accès à un tribunal est atteint dans sa substance même lorsque l'action en réparation est prescrite avant même que les victimes aient pu avoir objectivement connaissance de leurs droits. Ainsi, en l'espèce, un

106. CEDH, 28 oct. 1998, Osman c/R.-U., req. $\mathrm{n}^{\circ}$ 23452/94 ; contre exemple: CEDH, 19 oct. 2005, Roche c/R.-U., op. cit. ; CEDH, 14 déc. 2006, Markovic et al. c/Italie, op. cit.

107. CEDH, 9 déc. 1994, Les Saints-Monastères c/Grèce, série $\mathrm{A} \mathrm{n}^{0} 301$.

108. CEDH, 22 oct. 1996, Stubbings c/Royaume-Uni, req. $n^{\circ} 22083 / 93$ et $n^{\circ} 22095 / 93$. 
délai absolu de 10 ans voue à l'échec l'action des victimes de l'amiante, compte tenu de «la période de latence des maladies liées à l'exposition de l'amiante ${ }^{109} »$.

\subsection{Un accès au tribunal subordonné à l'exécution d'une décision antérieure}

En droit interne français l'obligation en matière pénale de se constituer prisonnier pour exercer une voie de recours a été censurée par la Cour de Strasbourg, cette dernière la considérant comme une atteinte disproportionnée au droit d'accès.

Dans une perspective analogue, le juge européen a considéré comme disproportionnée la déchéance du pourvoi en cassation d'un condamné qui n'a pas déféré au mandat d'arrêt décerné contre lui ${ }^{110}$.

Par ailleurs, en matière civile, doit être prise en compte la situation financière du justiciable, demandeur à un pourvoi en cassation, en cas $\mathrm{d}^{\prime}$ inexécution de la décision des juges du fond ${ }^{111}$.

Aux termes de ce survol par trop sommaire au regard de la richesse de la matière, il semble acquis que la reconnaissance du caractère fondamental du droit d'accès au juge a abouti, à l'exception de quelques limitations remarquables et parfois peu compatibles avec la notion de prééminence du droit, à une extension et à une intensification considérable de sa protection, au bénéfice du justiciable.

En particulier, il semble qu'on assiste à une certaine unification ou harmonisation des jurisprudences nationales et européennes en la matière, autour de la notion du procès équitable.

Dès lors, il est permis, au rebours de logiques managériales récentes, qui semblent avoir beaucoup inspiré les dernières réformes en droit interne, de souscrire à la thèse de certains auteurs selon laquelle cette évolution participe d'un mouvement plus général, outre de «fondamentalisation » du procès, de modélisation universelle de celui-ci, caractérisée par l'émergence de nouveaux principes directeurs, tels que la loyauté et le dialogue, qui transcenderaient l'ensemble des procédures.

Magistrat, professeur à l'université de Corse (2007-2015)

109. CEDH, 11 mars 2014, Howald Moor et al. c/Suisse, req. $n^{\circ}$ 52067/10, $\$ 74$.

110. CEDH, 23 nov. 1993, Poitrimol c/France, série A n ${ }^{\circ} 277-A, n^{\circ}$ 14032/88.

111. Voir art. 1009-1 du Code de procédure civile qui permet au premier président de la Cour de cassation de retirer du rôle le pourvoi d'un demandeur qui n'a pas exécuté la décision attaquée. 